\title{
Remote Sensing and Computational Epidemiology
}

\author{
Mohammad Reza Mobasheri
}

Remote sensing techniques have been developed over the past five decades and formed an important part of an interdisciplinary approach for many interdisciplinary science including health, environment, disease monitoring, biodiversity, and determination of habitat and ambient parameters. This new approach is based on the capability of looking at multispectral views of the environment at multiple spatial and temporal scales. This new interdisciplinary ideas of remote-sensing approach, have emerged for the detection, evaluation, and mapping of factors affecting public health. Such applications have helped to achieve considerable advancement in knowledge and insight for the environmental and public health administrations to work together in teams. This synergy has enabled them to explore solutions to previously unsolved environmental issues and managerial problems. Demand for talented researchers in remote sensing, GIS, and spatial modeling are continuously increasing. This includes environmental scientists, conservation, monitoring, and assessment experts. Perhaps it is not too much to hope that we can change our view of life and justify our habits to prevent what has happened during pandemic COVID-19 again. This book chapter is written with this hope in mind. Many scientists and resource managers already recognized the importance of adopting an approach in prediction of epidemics and pandemics before their occurrences.

\subsection{Introduction}

Remote sensing techniques have been developed over the past five decades and formed an important part of an interdisciplinary approach for many sciences including health, environment, disease monitoring, biodiversity, and determination of habitat and ambient parameters. The remote sensing technology is based on the capability of looking at multispectral views of the environment at multiple spatial and temporal scales. The information collected through this technology is readily integrated with other forms of data, including a global positioning system (GPS), geographical information system (GIS), and field observational data. This information is essential to prepare a foundation for species-specific models to map the habitat of any creatures as small as a virus and as large as an elephant. This may include testable predictions of their population dynamics and the development of biodiversity indicators and species-environment characteristics.

Interdisciplinary remote sensing consists of concepts, methodology, technology, and innovation. These elements when integrated may provide a unique opportunity to implement novel solutions to the problems that exist at the leading edge of environmental science and management. These problems are among the most complex issues of our time.

This interdisciplinary approach of remote-sensing has emerged for the detection, evaluation, and mapping of factors affecting public health. Such applications have helped to achieve considerable 
advancement in knowledge and insight for the environmental and public health administrations, enabling them to work together. This synergy has also enabled them to explore solutions to previously unsolved environmental issues and managerial problems.

There are increasing belief and evidence that the health of human being and other species is adversely affected by human activities and landscape change [1-3]. Perhaps it is not too much to hope that we can change our view of life and justify our habits to prevent what has happened during pandemic COVID-19 again.

Interdisciplinary researches to understanding the environmental factors for the insects to be prepared for breeding and extraction of these factors from satellite images have shown the power of remote sensing in monitoring environmental issues. Of course not sufficient mainstream remote-sensing research texts have dealt with this emerging approach in detail to help the growing collaboration among those specializing in remote sensing, health management, and ecosystem scientists, in critical decision making and efforts on the ground. This book chapter is written with this hope in mind. Many scientists and resource managers have already recognized the importance of adopting an approach in prediction of epidemics and pandemics before their occurrences [4, 5], and in parallel with this recognition, the use of remote sensing and GIS approaches have noticeably increased [6, 7]. For example, research carried by Ahmadian et al. [4], regarding Malaria outbreak, has proved the potential of remote sensing and GIS. A section of this chapter is assigned to this work. Also, a section is assigned to Cholera epidemic prediction. Finally, an approach to the prediction of COVID-19 epidemic occurrence using remote sensing and surface data is suggested.

\subsection{Remote Sensing and Health}

In what follows, remote sensing technology is discussed. This is followed by an explanation on how this technology is related to the virus and some other vector disease.

\subsubsection{What is a Virus?}

A virus is a submicroscopic infectious agent that replicates itself only inside the living cells of an organism. Viruses can infect all types of life forms, from animals and plants to microorganisms, including bacteria and archaea [8]. Viruses are found in almost every ecosystem on Earth and are the most numerous type of biological entity $[9,10]$. When infected, a host cell is forced to rapidly produce thousands of identical copies of the original virus. Most virus species have virions too small to be seen with an optical microscope as they are one hundredth the size of most bacteria.

Viruses spread in many ways. One transmission pathway is through disease-bearing organisms known as vectors: for example, viruses are often transmitted from plant to plant by insects that feed on plant sap, such as aphids; and viruses in animals can be carried by blood-sucking insects and vampire bats. The infectious dose required to produce infection in humans is less than 100 particles in Influenza viruses $[11,12]$ and to some extent in COVID-19. The variety of host cells that a virus can infect is called its "host range". This can be narrow or broad, meaning a virus is capable of infecting only few species, or infecting many.

Viral infections in animals provoke an immune response that usually eliminates the infecting virus. Immune responses can also be produced by vaccines, which confer an artificially acquired immunity to the specific viral infection. Some viruses, including those that cause AIDS, HPV infection, viral hepatitis, and COVID-19 evade these immune responses and result in chronic infections.

\subsubsection{How is a Virus related to Remote Sensing?}

In this section, the relation between different respiratory diseases (including COVID-19) and the atmospheric and environmental parameters that are investigated by different workers will be 
discussed. All these atmospheric and environmental parameters can be assessed by and extracted from images acquired by different sensors on board of different satellites.

Many researchers have observed a connection between the occurrence of respiratory diseases such as influenza (A and B) and COVID-19 and climatic parameters such as air temperature, air moisture content, relative humidity, wind speed, and amount of precipitation $[12,13]$. Although the outbreak and epidemic, contagious on person to person, the weather conditions may play a key role in making some regions potentially suitable for the virus to be activated (Ianevski et al. 2019). This is the case for many other epidemic diseases such as Malaria and Cholera. In what follows, a few of these researches will be introduced.

Peci et al. [13], claimed that the occasion of incidence of influenza increases during rainy seasons in tropical climates and during the dry, cold months of winter in temperate climates. They found that the seasonality of influenza $\mathrm{A}$ and $\mathrm{B}$ viruses is different in most temperate climates. A negative association of both absolute humidity and temperature with influenza A and B viruses was observed.

Guo et al. [12] found an association between mean temperature, relative humidity ( $\mathrm{RH})$, and the atmospheric pressure with influenza cases for children between 0-17 years old. They claimed that the relative risks increase as the temperature falls below $20^{\circ} \mathrm{C}, \mathrm{RH}$ lower than $50 \%$, or higher than $80 \%$. Also, the risk of influenza increased with rising atmospheric pressure with $1,005 \mathrm{hPa}$ as the breakpoint. They found that the effect of coldness, humidity, dryness, high-pressure, and low-pressure showed statistical significance both in females and males. The cold effect increases with age while the humidity affected all ages of children, whereas, dryness mainly affects ages 4-14 years. High-pressure mainly affected the age of 0-3, whereas the low-pressure affects preschool children aged 0-6 years old.

Ianevski et al. [14] investigated the effects of meteorological parameters such as temperature, UV index, humidity, wind speed, atmospheric pressure, and precipitation (all acquirable by remote sensing technique) on IV activity in Norway, Sweden, Finland, Estonia, Latvia, and Lithuania during 2010-2018 in Influenza virus epidemics while considering the pace of global warming. They deployed correlation and machine learning analysis techniques and found that low temperature and UV radiation can preserve Influenza virus infectivity. The researchers believe that low temperature and UV index were the most suitable predictive indexes among other meteorological factors in Northern Europe. Of course, their in-vitro experiments confirmed that low temperature and UV radiation preserved Influenza virus infectivity.

Most recently, Wang et al. [15] have researched the ongoing global pandemic of COVID-19. Their aim was to predict the effect of the upcoming summer in the northern hemisphere and expected to have a reduction of the transmission intensity of COVID-19 with increasing humidity and temperature. They used data from the cases with symptom-onset dates from January 19 to February 10, 2020, for 100 Chinese cities, and cases with confirmed dates from March 15 to April 25 for 1,005 U.S. counties. The relationship between the transmissibility of COVID-19 and the temperature/humidity was assessed. They found a similar influence of the temperature and relative humidity on effective reproductive number ( $\mathrm{R}$ values) of COVID-19 for both China and the U.S. before lockdown in both countries. There was the reduction of reproduction by increasing temperature and humidity. This reduction of transmission was not further continued until July when the temperature and humidity had risen.

In an unpublished work of $\mathrm{Xu}$ et al. [16], the impact of environmental factors including pollution contaminants on COVID-19 transmission was investigated. They studied the relative risk of COVID-19 due to weather conditions and ambient air pollution. In this work, the daily reproduction at 3,739 global locations was controlled for the delay between infection and detection. After that they associated these with local weather conditions and ambient air pollution. They observed a negative relationship between the estimated reproduction number and temperatures above $25^{\circ} \mathrm{C}$, and a U-shaped relationship with outdoor ultraviolet exposure, with a weaker positive association with air pressure, wind speed, precipitation, diurnal temperature, $\mathrm{SO}_{2}$, and ozone.

This was followed by a projection of the relative risk of COVID-19 transmission due to environmental factors in 1,072 global cities. The findings showed that warmer temperature and moderate outdoor ultraviolet exposure may offer a modest reduction in transmission.

Scafetta [17] investigated about COVID-19 and its possible relation to specific weather conditions. The findings showed that the 2020 winter weather in the region of Wuhan (Hubei, 
Central China) - where the virus first showed up in December 2019 and spread widely from January to February 2020 was very similar to that of the Northern Italian provinces of Milan, Brescia, and Bergamo, where the pandemic has been very severe from February to March 2020. According to this study such similarity may suggest the worsening of the pandemic under weather temperatures between $4^{\circ} \mathrm{C}$ and $11^{\circ} \mathrm{C}$. Based on this result, Scafetta [17] prepared maps of world-specific isotherm to locate, month by month, the world regions that share similar temperature ranges. The analysis showed that this isotherm zone extended mostly from Central China toward Iran, Turkey, West-Mediterranean Europe (Italy, Spain, and France) up to the United State of America from January to March 2020, and coinciding with the geographic regions most affected by the pandemic in the same period. He predicted that in Autumn of the Northern hemisphere, the pandemic could return and affect the same regions again. Scafetta [17] believes that the Tropical Zone and the entire Southern Hemisphere, but in restricted southern regions, could avoid a strong pandemic because of the sufficiently warm weather during the entire year.

In a study, Njifon et al. [18] investigated the role of meteorological parameters in the seasonality of influenza viruses in tropical and subtropical regions particularly in Northern Cameroon, a region characterized by high temperatures. The researchers focused on the effect of temperature, humidity, and rainfall from January 2014 to December 2016. Their results and conclusion analysis showed that there was a statistically profound association between overall influenza activity and influenza A activity for average relative humidity. They noticed a lag between humidity rise and Influenza activity where a unit increase in humidity within a given month leads to more than $85 \%$ rise in overall influenza and influenza A activity two months later. However, they believed that none of the three meteorological variables could explain the influenza B activity.

Besides the dependence of Influenza and COVID-19 to the weather parameters, there are other vector-borne diseases such as Malaria, Cholera, Ebola, and Dengue that all proved to be dependent on some weather parameters and environmental conditions. Ahmadian et al. [4], conducted a research for the determination of high potential region for Malaria outbreak using satellite images. The findings of this research showed that the insect begins breeding when the temperature is between $25 \mathrm{C}$ and $35^{\circ} \mathrm{C}$, relative humidity between 50 to $80 \%$, presence of vegetation cover, and presence of water pools. All these factors were supplied using Landsat images and overlaid to find the risk potential area. The results were compared with the number of registered patients in all sentinels around within the study area. Details of the methodology are presented in the following section.

\subsection{Remote Sensing Methods to Predict Health-related Outbreaks}

This section is assigned to the remote sensing methods by which disease epidemic and outbreaks regions can be predicted. Numerous research has so far been conducted to predict time and regions where a disease outbreak or epidemic occurs.

\subsubsection{Malaria Case Study}

Based on WHO [19], in 2017, an estimated 219 million cases of malaria occurred worldwide, compared with 239 million cases in 2010 and 217 million cases in 2016. Although there were an estimated 20 million fewer malaria cases in 2017 than in 2010, data for the period 2015-2017 highlight that no significant progress in reducing global malaria cases was made in this time frame. Most malaria cases in 2017 were in the WHO African Region (200 million or 92\%), followed by the WHO South-East Asia Region with 5\% of the cases and the WHO Eastern Mediterranean Region with $2 \%$.

Malaria is an infectious disease that is being transferred by the female mosquito of the species Anopheles. Out of four malaria parasites responsible for disease outbreak, Plasmodium falciparum is the most important one. The life cycle of the malaria parasite develops in the anopheline and in the human body [20, 21]. 
These parasites require suitable environmental parameters to complete their development cycle within the mosquito. These parameters are temperature, humidity, vegetation, and water [4]. The cycle begins when the anopheline sucks human blood usually during the night time. After two to three days the mosquito starts breeding. The breeding sites are usually water, preferably swamps or slow-flowing water bodies. In the dry and semi-dry regions, these water bodies can appear after the occurrence of heavy seasonal rain in the region. Depending on the parasite species and availability of suitable humidity and air temperature, it takes 8 to 30 days for the parasite to develop in the mosquito after which the parasitic will be ready to transmit to humans through mosquito stings.

The influence of the temperature on the feeding behavior of the mosquito, its survival, and the length of the cycle that the parasite needs to develop in the mosquito before it can be infective to humans is immense. For instance, the main mosquito species responsible for Plasmodium falciparum infections feed every second day at $25^{\circ} \mathrm{C}$ and every third day at lower temperatures [21].

The chance of mosquito survival is low at extreme temperatures i.e. the temperatures below $5^{\circ} \mathrm{C}$ and temperatures above $40^{\circ} \mathrm{C}$ (in some reports $35^{\circ} \mathrm{C}$ ) are deadly for the mosquito. In this regard, the optimum mosquito survival chance is found at $32^{\circ} \mathrm{C}$ [21].

The environmental vulnerability of the disease is mainly determined by the effects that climatic factors can have on the abundance of malaria vectors. The population of vectors depends critically upon elements of the weather and land-use all achievable using remote sensing data [22-24]. Then epidemiological and demographic models can be deployed to relate these data to estimate the distribution of humans and parasites at a high spatial resolution [22]. Such models can in turn help in providing an empirical basis for defining the disease burden of polyparasitism and the potential health impact of removing or reducing disease risk.

In summary, a temperature range of $25^{\circ} \mathrm{C}$ to $35^{\circ} \mathrm{C}$ and a relative humidity range of 50 to 80 percent is suitable for developing malaria outbreaks. In a study conducted by Ahmadian et al. [4], a methodology for extracting temperature, humidity, water bodies, and vegetated area all from satellite images is presented and details of which are as follows.

\subsubsection{Materials and Methods}

In the work of Ahmadian et al. [4], when needed, weather data collected in nearby synoptic stations were used as ground truth. Also the synoptic station reports were used to select the proper satellite images after raining occurrence. Analysis of the weather stability and visibility for estimation of the severity of the atmospheric effects on the satellite images were two other important aspects of using weather data.

The Satellite images used in this work were selected upon these criteria:

- The image should contain regions where the malaria outbreaks occur

- The image should contain regions where a considerable amount of raining has happened in one to a few days before the date of image acquisition

- The visibility in the regions covered by the image scene should be greater than 10 kilometers

- The region should contain a minimum amount of cloud cover

- The image acquisition date should be between August to November and between February to May when most of the raining in the region happens

- The sensors having thermal bands were used for LST calculation

- The availability of malaria statistics for the period of the study

Taking all these conditions into account, only Landsat and ASTER images were found suitable where the former images were available. Out of five Landsat images from November 2000 to August 2005, the image of April 19, 2003 was found the most suitable one for this study. Noting that all Landsat images with acquisition dates beyond April 2003 were defected due to the malfunctioning of the 7ETM+ Scan Line Corrector [22, 25].

Geo-referencing, Radiometric calibration (Atmospheric corrections), and DN to radiance and 
then reflectance conversion for the visible and SWIR and DN to radiance for thermal bands were the processing steps that were taken. These images were used for producing the following products.

\subsubsection{Study area}

The study area was two districts located in the south of Iran right at the north of Hormuz straight in the Persian Gulf (Figure 4.1). The two districts are Minab and Kahnooj. Minab is situated between 26, 7' $\mathrm{N}$ and $27,26^{\prime} \mathrm{N}$ and 56, 48' $\mathrm{E}$ and 57, 50' $\mathrm{E}$ with an altitude of $27 \mathrm{~m}$ from mean sea level and approximately 104 kilometers from the Persian Gulf. Kahnooj on the other hand is located between $27,50 ' \mathrm{~N}$ and $28,59^{\prime} \mathrm{N}$ and $56,45^{\prime} \mathrm{E}$ to $57,53^{\prime} \mathrm{E}$ with an altitude of $469 \mathrm{~m}$ from mean sea level approximately 330 kilometers inland. The malaria outbreaks data for these two districts were collected from the related Health and Hygiene Bureau (Table 4.1).

\section{TABLE 4.1}

Number of affected people in monthly malaria outbreaks for the period of 2003-2004

\begin{tabular}{ccccccccccccc}
\hline Month & Apr & May & Jun & Jul & Aug & Sep & Oct & Nov & Dec & Jan & Feb & Mar \\
\hline Minab & 3 & 17 & 27 & 15 & 6 & 380 & 1020 & 339 & 95 & 17 & 1 & 8 \\
Kahnooj & 13 & 40 & 23 & 45 & 35 & 20 & 11 & 28 & 5 & 0 & 4 & 4 \\
\hline
\end{tabular}

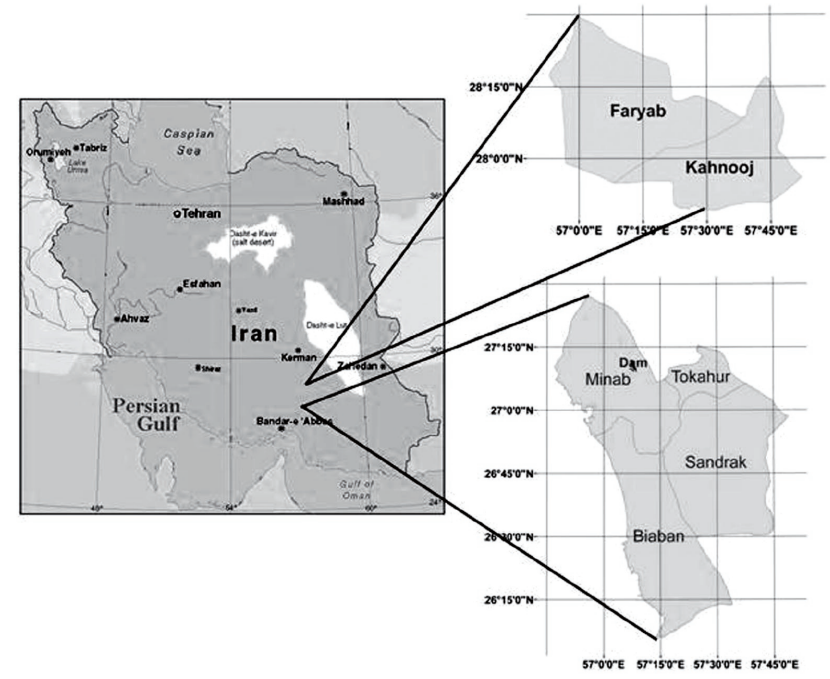

FIGURE 4.1

Location of the study area

\subsubsection{Malaria Distribution Maps for Incidence Factors}

Usually, three indices of Prevalence, API, and Incidence are being used in malaria outbreak investigations where the Incidence index is used in this research. The reason for this selection is the short duration required for the calculation of this index [4]. This index can be calculated using equation (4.1):

$$
\text { Incidence }=\frac{M}{P_{\text {month }}} \times k
$$

where $\mathrm{M}$ is the number of positive cases in a few months, $P$ is the average population in the region 
for those months, and $\mathrm{k}$ is a scale factor equal to 1,000. Table 4.2 shows the health centers, region population, and calculated Incidence index for Minab and Kahnooj region respectively.

\section{TABLE 4.2}

Health centers, region population, and calculated Incidence index for Minab (left) and Kahnooj (right)

\begin{tabular}{|c|c|c|c|c|c|}
\hline $\begin{array}{l}\text { Health } \\
\text { Center }\end{array}$ & $\begin{array}{c}2003 \\
\text { Population }\end{array}$ & $\begin{array}{c}\text { Incidence } \\
\text { April to June }\end{array}$ & $\begin{array}{l}\text { Health } \\
\text { Center }\end{array}$ & $\begin{array}{c}2003 \\
\text { Population }\end{array}$ & $\begin{array}{c}\text { Incidence } \\
\text { April to June }\end{array}$ \\
\hline Darpahn & 6,702 & 0.149 & City Zone 1 & 12,452 & 2.811 \\
\hline Sandark & 13,429 & 0.074 & City Zone 2 & 11,677 & 2.569 \\
\hline Sareni & 11,481 & 0 & City Zone 3 & 13,048 & 0.077 \\
\hline Hashtbandi & 11,481 & 0.087 & Sahlavar & 5,340 & 0.187 \\
\hline Karian & 13,665 & 0.073 & Chah Morid & 5,217 & 0.575 \\
\hline Banzark & 25,190 & 0.198 & Hoorani & 16,455 & 0.425 \\
\hline Sirik & 12,439 & 0.241 & Faryab & 11,631 & 2.493 \\
\hline Bemani & 21,450 & 0 & Dehpish & 5,065 & 0.395 \\
\hline Hakami & 14,305 & 0.070 & & & \\
\hline Tiab & 10,787 & 0.185 & & & \\
\hline Haj Khademi & 64,807 & 0.015 & & & \\
\hline Minab City & 62,652 & 0.303 & & & \\
\hline
\end{tabular}

\subsection{Vegetated Area Mapping}

To map the vegetated area in the images, usually vegetation indices such as Normalized Difference Vegetation Index (NDVI), Simple Ratio (SR), and Enhanced Vegetation Index (EVI) or modified version of them is being used. The index used in this work was NDVI where the threshold value of 0.2 was used. This threshold was tested using ground data and other vegetation indices. A detailed discussion regarding uncertainties involved with this threshold value will be presented in the discussion and analysis section. The NDVI index takes advantage of the red edge effect in the vegetation spectral reflectance curve i.e. low reflectance in the red $\rho_{\mathrm{R}}$ and very high reflectance in near-infrared $\rho_{\text {NIR }}$. The reflectance difference in these two spectral bands can be normalized to 1 using the following equation:

$$
N D V I=\frac{\rho_{\mathrm{NIR}}-\rho_{\mathrm{R}}}{\rho_{\mathrm{NIR}}+\rho_{\mathrm{R}}}
$$

NDVI varies between -1 and +1 where its value for vegetated pixels is generally greater than 0.2 , with values exceeding 0.65 indicating dense vegetation. Taking a flying range of mosquitoes into account a two kilometers distance around any vegetated point is considered.

\subsection{Water Body Mapping}

There are different methods and approaches for mapping patches of water bodies in the scenes where Normalized Difference Water Index (NDWI) and Tasselled Cap Transformation (TC) are two appropriate ones. Using field data, they found TC more appropriate for this work. This transformation takes advantage of 6 bands of $7 \mathrm{ETM}+$ sensor and is of the form [26]: 


$$
\left[\begin{array}{c}
\text { Brightness } \\
\text { Greenness } \\
\text { Wetness }
\end{array}\right]=\left[\begin{array}{cccccc}
b 1 & b 2 & b 3 & b 4 & b 5 & b 7 \\
g 1 & g 2 & g 3 & g 4 & g 5 & g 7 \\
w 1 & w 2 & w 3 & w 4 & w 5 & w 7
\end{array}\right] \times\left[\begin{array}{l}
C H 1 \\
C H 2 \\
C H 3 \\
C H 4 \\
C H 5 \\
C H 7
\end{array}\right]
$$

where $\mathrm{CH} 1$ to $\mathrm{CH} 7$ stands for reflectance in bands 1 to 7 (excluding band 6 which is thermal). bi, gi, and wi are coefficients of transformation for brightness, greenness, and wetness respectively, and are shown in Table 4.3 .

\section{TABLE 4.3}

Tasseled cap transformation coefficients for 7ETM+ sensor

\begin{tabular}{crrrrrr}
\hline Bands & 1 & 2 & 3 & 4 & 5 & 7 \\
bi & 0.3561 & 0.3972 & 0.3904 & 0.6966 & 0.2286 & 0.1596 \\
gi & -0.3344 & -0.3544 & -0.4556 & 0.6966 & -0.0242 & -0.2630 \\
wi & 0.2626 & 0.2144 & 0.0926 & 0.0656 & -0.7629 & 0.5388 \\
\hline
\end{tabular}

For the regions containing patches of the water body, wetness takes a value of greater than -0.0710. Applying this threshold to the image and again taking 2 kilometers flight zone for the mosquitoes around water bodies, figures 8 and 9 for Minab and Kahnooj were produced respectively. The detected water bodies in the Kahnooj image were as small as a pixel and consequently cannot be detected visually in the figure.

\subsection{Land Surface Temperature}

To calculate LST, the following equation suggested by the Landsat team is used:

$$
T_{s}=\frac{k_{2}}{L_{n}\left(\frac{\epsilon_{N B} k_{1}}{R_{c}}+1\right)}
$$

where $R_{c}$ is the corrected spectral flux density that reaches the sensor $\left(\mathrm{W} / \mathrm{m}^{2} / \mu \mathrm{m}\right), \varepsilon_{\mathrm{NB}}$ is the surface narrow-band emissivity, $k_{1}$ and $k_{2}$ are constants equal to 666.09 (Kelvin) and 1282.71 $\left(\mathrm{W} / \mathrm{m}^{2} / \mu \mathrm{m}\right)$ respectively [27]. Applying equation (4.4) to the channel 6 radiance image, the surface temperature for Minab and Kahnooj was calculated.

\subsection{Air Temperature}

To calculate air temperature, the Surface Energy Balance Algorithm for Land (SEBAL) is used [28]. This algorithm consists of 25 modules where a combination of empirical formulas, synoptic data from nearby weather stations, and image extracted data (such as surface temperature) are being processed in these modules. Detail of this algorithm can be found in [28]. The SEBAL algorithm output maps for air temperature for Minab and Kahnooj are shown while the temperature range of $25^{\circ} \mathrm{C}$ to $35^{\circ} \mathrm{C}$ is imposed on the image (Figure 4.2). It can be seen that except in very small areas, the other parts of the region have temperature within the $25^{\circ} \mathrm{C}$ to $35^{\circ} \mathrm{C}$ zone. 


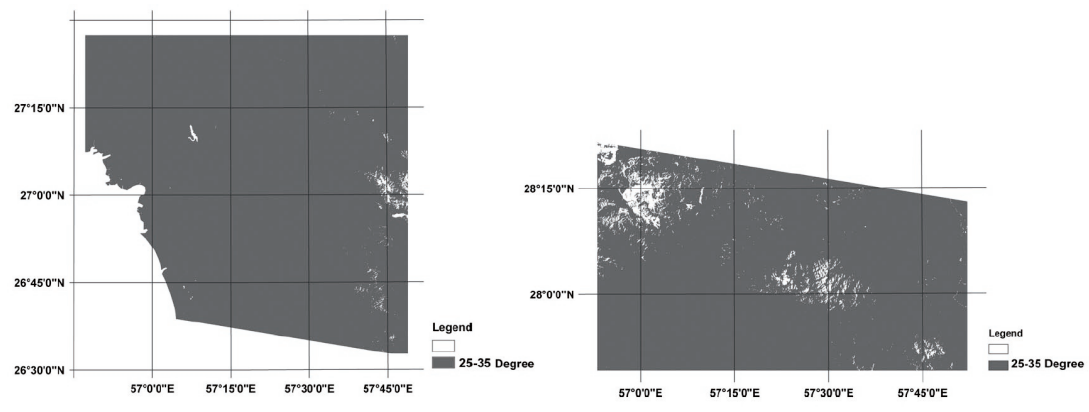

\section{FIGURE 4.2}

Satellite extracted maps of air temperature between $25^{\circ} \mathrm{C}$ and $35^{\circ} \mathrm{C}$ for Minab (left) and Kahnooj (right)

\subsection{Relative Humidity}

Using the calculated air temperature image in the previous step one can calculate the saturated partial water vapor pressure using the following equation [4]:

$$
e_{s}=6.1121 \times \exp \left[\left(17.502 \times T_{a}\right)\left(240.97+T_{a}\right)^{-1}\right]
$$

where $e_{s}$ is saturated partial water vapor pressure in millibar and $T_{a}$ is the air temperature in degrees of Celsius. Then using partial vapor pressure $e$ calculated from measured synoptic station data and assuming negligible horizontal gradient in $e$ for the time of image acquisition, the relative humidity $R H$ in the selected scene can be calculated using equation (4.6) below:

$$
R H=e \times 100
$$

They produced maps of relative humidity (for $50 \%$ to $80 \%$ range).

\subsection{Results and Analysis}

Now all maps of parameters necessary for high-risk region determination are prepared. Since these parameters do not affect the mosquito's survival equally, then a simple overlying of these layers of information would not be appropriate and parameters such as temperature, humidity, water body, and vegetation must have different weighting coefficients. An unpublished work of suggested weighing coefficients of $0.35,0.25,0.25$, and 0.15 for temperature, humidity, water body, and vegetation respectively. Having overlaid these layers using appropriate weighing coefficients, the resulted high-risk map is shown in Figure 4.3 where number 10 shows the highest risk.

Combining the incidence indices (Section 4.3.4) and Figure 4.3 helps compare between different calculated risk regions and calculated incidence index using field data (Figure 4.4). As can be seen, the highest risk regions can only be found in Minab. This degree of risk cannot be seen in Kahnooj mostly due to the lack of suitable humidity conditions. 


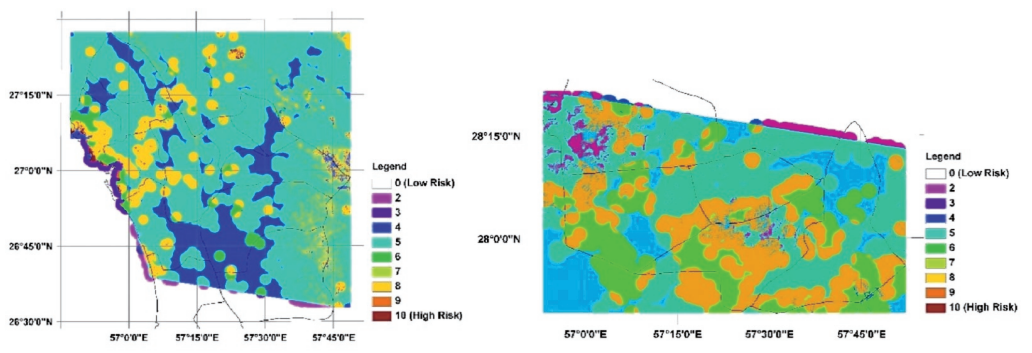

FIGURE 4.3

High-risk maps of Minab (left) and Kahnooj (right). Higher numbers represent higher risk regions

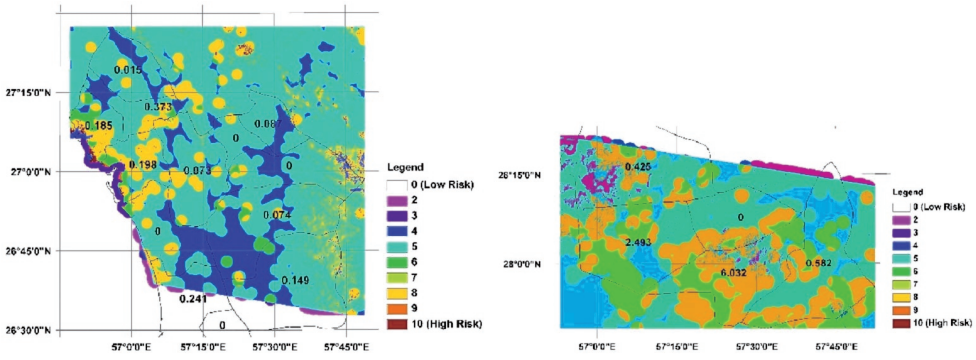

\section{FIGURE 4.4}

Comparison of different calculated risk regions (Figure 4.3) and calculated incidence index using field data. The zeros in the image are due to the lack of information for those regions.

\subsection{Discussion}

There are sources of uncertainties with the extraction of any layers of information from satellite images. The amount of uncertainties compared to the field data differ for different parameters. The uncertainties are due to the unknown surface emissivity values for LST extraction of up to $2^{\circ} \mathrm{C}$, uncertainties in relative humidity of up to $3 \%$ due to the assumption of uniformity for partial vapor pressure throughout the sub-scene, and the uncertainty in the air temperature. The uncertainties in vegetation and water bodies mapping are of the second order of importance because these uncertainties can be included in the 2 kilometers flying zone already considered in the maps. Adding the uncertainties in LST and relative humidity to the lower and upper limits of temperature and humidity, the effect found was a small expansion around the potential area. Since this research aimed to show the high-risk potential area to the local manager for deputing mosquitoes exterminating group to these regions, a few meters away from the high-risk region will not make much difference.

It is found that one can predict the malaria outbreak by extracting environmental parameters necessary for mosquito's parasite life cycle. These parameters were temperature $\left(25^{\circ} \mathrm{C}\right.$ to $\left.35^{\circ} \mathrm{C}\right)$, relative humidity ( $50 \%$ to $80 \%$ ), patches of water, and vegetation covers. Regarding 10 degrees in temperature range and $30 \%$ in humidity range, as well as 2 kilometers flying zone for the mosquito, any intrinsic uncertainties in the extraction of these parameters from satellite images due to their resolutions and intervening atmosphere - which were the sources of concern for many satellite approaches - did not affect the results seriously. A weighed overlying of the four layers of information i.e. air temperature, relative humidity, patches of water bodies, and vegetated area showed acceptable agreements with the field-collected data. Of course, there are still some uncertainties involved with the weighing coefficients for these layers which call for further investigation. Also, there are other parameters such as shadows, manmade pools, pots of water, 
and many other factors that may help and affect the outbreaks which each must be considered separately. However, the satellite ability in the detection of high-risk potential regions may provide non-expensive information on a routine basis not only in malaria but for other epidemics as well, where studies in those areas are the aims of these authors.

\subsection{Cholera Case Study}

Vibrio Cholerae (VC), a bacterium autochthonous to the aquatic environment, is the agent causing Cholera, a severe aquatic, life-threatening diarrheal disease occurring mostly in developing countries. VC has many different types of serogroups, only two of which can cause epidemic cholera. Those two serogroups are called serogroups O1 and serogroups O139 (O139 is found only in Asia) and can cause epidemic cholera if they also produce the cholera toxin. VC, including both serogroups $\mathrm{O} 1$ and $\mathrm{O} 139$, is found in association with crustacean zooplankton, mainly copepods, and notably in ponds, rivers, estuarine, and coastal region globally. Cholera bacteria attach to zooplanktons (copepods), form thin biofilms in the brackish water especially in coastal regions. Since copepods feed on phytoplankton, the proliferation of phytoplankton increases the number of cholera bacteria. (Shafiqul Islam), a member of an interdisciplinary research team from Tufts University and National Oceanic and Atmospheric Administration, US. The incidence of cholera and the occurrence of pathogenic VC strains with zooplankton were studied in many areas.

If one can measure the density of chlorophyll and track the blooming of phytoplankton, the prediction of Cholera outbreak (mostly in endemic regions) seems possible. The satellite data on chlorophyll concentration could be used to routinely monitor coastal processes and track cholera outbreaks. The privilege of using satellite data is its suitable spatial and temporal coverage as well as low expenses.

Rahimi-Doab [5] conducted research on Cholera endemic regions. He claimed that the bloom or the flowering of phytoplankton, and as a result, the flourishing of the zooplankton and feeding are closely correlated with the temperature at the sea surface (SST). The SST can be continuously monitored through thermal channels of satellite sensors. By monitoring SST and measuring the seawater chlorophyll as a sign of plankton flourishing and knowing the amount of rainfall as an agent for the transfer of micronutrient materials from shore to sea, Rahimi-Doab (2018) could predict the disease outbreak one month in advance. However, the disease has its trend in each region and the peak of the disease in each region was different in terms of time and number of patients. This requires calibrating the suggested model for each region. In Rahimi-Doab's [5] work, the statistical analysis of the log-linear Poisson Generalized Linear Model (GLM) was used to predict the appropriate time for the growth of $\mathrm{VC}$ in the sea and its subsequent outbreak. For this purpose, the effects of three environmental factors i.e. SST, chlorophyll concentration of seawater extracted from the products of MODIS onboard of Aqua platform, and the rainfall data supplied by TRMM satellite, were all taken as independent variables and investigated. The case study region was the 2011 outbreak in Benin, Africa and the results showed that these factors were correlated to the disease outbreak in that endemic region.

Finally, the model was applied to the outbreak cases in the southern coastal regions of Iran. The model could predict the occurrence of the outbreak, one month in advance. Although the output of the model tells us the number of patients however, this number depends on the suitability of each of the three environmental factors, which are included as independent variables in the model.

\subsection{Conclusions}

The two aforementioned case studies showed that the environmental factors and parameters are playing a key role in the occurrence of outbreak and/or epidemic in many cases. This includes 
vector-borne diseases as well as viruses including COVID-19. All these environmental parameters are acquirable from satellite images. As mentioned in previous sections, COVID-19 and other similar viruses have shown some dependencies on some environmental parameters but, the main issue in these kinds of epidemics is the behavior of the patients affected or those who carry the viruses and spread it in the ambient. Our preliminary studies show that we might be able to classify different parts of the city based on the temperature, humidity, wind speed and direction, surface cover, height, and air pressure, and some other factors and compare these classes with the results of the affected people's population in those regions. The equation that could be the basis of this investigation can be of the form of equation (4.7):

$$
C O V I D-C F=f(T a, L S T, R H, P, V s, V d, S C, H)
$$

Where COVID - CF, Ta, LST, RH, P, Vs, Vd,SC, and $H$ are COVID-19 Contagious Factor, air temperature at $2 \mathrm{~m}$ height, Land Surface Temperature, Relative Humidity, air pressure, wind speed, wind direction, Surface Cover, and Height of the land on that region, respectively.

The work which is currently undergoing showed the dependence of the Influenza and to some extent COVID-19 outbreaks to the air temperature, humidity, wind speed and direction, and density of the population. The results, which will be published separately, may help to highlight the hotspots and inform the people regularly.

\section{References}

[1] P. Daszak, A.A. Cunningham, and A.D. Hyatt. Anthropogenic environmental change and the emergence of infectious diseases in wildlife. Acta Tropica, 78(2):103 - 116, 2001. ISSN 0001-706X. doi: https://doi.org/ 10.1016/S0001-706X(00)00179-0.

[2] S.K. Majumdar. Wildlife Diseases: Landscape Epidemiology, Spatial Distribution and Utilization of Remote Sensing Technology. Book publications of the Pennsylvania Academy of Science. Pennsylvania Academy of Science, 2005. ISBN 9780945809197.

[3] Daniel B. Botkin, Henrik Saxe, Miguel B. Araújo, Richard Betts, Richard H. W. Bradshaw, Tomas Cedhagen, Peter Chesson, Terry P. Dawson, Julie R. Etterson, Daniel P. Faith, Simon Ferrier, Antoine Guisan, Anja Skjoldborg Hansen, David W. Hilbert, Craig Loehle, Chris Margules, Mark New, Matthew J. Sobel, and David R. B. Stockwell. Forecasting the Effects of Global Warming on Biodiversity. BioScience, 57(3): 227-236, 03 2007. ISSN 0006-3568. doi: 10.1641/B570306.

[4] A. Ahmadian Marj, M. R. Mobasheri, M. J. Valadan Zoej, Y. Rezaei, and M. R. Abaei. Exploring the use of satellite images in the estimation of potential malaria outbreak regions. Environmental Hazards, 8(2): 89-100, 2009. doi: 10.3763/ehaz.2009.0003.

[5] M Rahimi-Doab. Cholera Outbreak Potential Region Determination Using Satellite Images. Master's thesis, Khavaran Institute of Higher Education, 2018.

[6] R.I. Miller. Mapping the Diversity of Nature. Springer Netherlands, 1994. ISBN 9780412455100.

[7] Woody Turner, Sacha Spector, Ned Gardiner, Matthew Fladeland, Eleanor Sterling, and Marc Steininger. Remote sensing for biodiversity science and conservation. Trends in Ecology \& Evolution, 18(6):306-314, 2003. ISSN 0169-5347. doi: https://doi.org/10.1016/S0169-5347(03)00070-3.

[8] Eugene V. Koonin, Tatiana G. Senkevich, and Valerian V. Dolja. The ancient virus world and evolution of cells. Biology Direct, 1:29-29, Sep 2006. ISSN 1745-6150. doi: 10.1186/1745-6150-1-29.

[9] C. Martin Lawrence, Smita Menon, Brian J. Eilers, Brian Bothner, Reza Khayat, Trevor Douglas, and Mark J. Young. Structural and functional studies of archaeal viruses. The Journal of biological chemistry, 284(19): 12599-12603, May 2009. ISSN 0021-9258. doi: 10.1074/jbc.R800078200.

[10] Robert A Edwards and Forest Rohwer. Viral metagenomics. 3(6):504-510, June 2005. doi: $10.1038 /$ nrmicro1163.

[11] Elizabeth Robilotti, Stan Deresinski, and Benjamin A. Pinsky. Norovirus. Clinical Microbiology Reviews, 28 (1):134-164, Jan 2015. ISSN 1098-6618. doi: 10.1128/CMR.00075-14.

[12] Qiaozhi Guo, Zhiqiang Dong, Weilin Zeng, Wenjun Ma, Danyang Zhao, Xin Sun, Sitang Gong, Jianpeng Xiao, Tiegang $\mathrm{Li}$, and Wensui $\mathrm{Hu}$. The effects of meteorological factors on influenza among children in guangzhou, china. Influenza and other Respiratory Viruses, 13(2):166-175, Mar 2019. ISSN 1750-2659. doi: $10.1111 /$ irv.12617. 
[13] Adriana Peci, Anne-Luise Winter, Ye Li, Saravanamuttu Gnaneshan, Juan Liu, Samira Mubareka, and Jonathan B. Gubbay. Effects of Absolute Humidity, Relative Humidity, Temperature, and Wind Speed on Influenza Activity in Toronto, Ontario, Canada. Applied and Environmental Microbiology, 85(6):e02426-18, Mar 2019. ISSN 1098-5336. doi: 10.1128/AEM.02426-18.

[14] Aleksandr Ianevski, Eva Zusinaite, Nastassia Shtaida, Hannimari Kallio-Kokko, Miia Valkonen, Anu Kantele, Kaidi Telling, Irja Lutsar, Pille Letjuka, Natalja Metelitsa, Valentyn Oksenych, Uga Dumpis, Astra Vitkauskiene, Kestutis Stašaitis, Christina Öhrmalm, Kåre Bondeson, Anders Bergqvist, Rebecca J. Cox, Tanel Tenson, Andres Merits, and Denis E. Kainov. Low temperature and low uv indexes correlated with peaks of influenza virus activity in northern europe during 2010-2018. Viruses, 11(3):207, Mar 2019. ISSN 1999-4915. doi: 10.3390/v11030207.

[15] Jingyuan Wang, Ke Tang, Kai Feng, and Weifeng Lv. High temperature and high humidity reduce the transmission of covid-19. SSRN Electronic Journal, 2020. doi: 10.2139/ssrn.3551767.

[16] Ran Xu, Hazhir Rahmandad, Marichi Gupta, Catherine Digennaro, Navid Ghaffarzadegan, Heresh Amini, and Mohammad S. Jalali. The Modest Impact of Weather and Air Pollution on COVID-19 Transmission. SSRN Electronic Journal, 2020. doi: 10.2139/ssrn.3593879.

[17] Nicola Scafetta. A Proposal for Isotherm World Maps to Forecast the Seasonal Evolution of the SARS-CoV-2 Pandemic. Preprints, (2020040063), 2020. doi: 10.20944/preprints202004.0063.v1.

[18] Hermann Landry Munshili Njifon, Chavely Gwladys Monamele, Cyprien Kengne Nde, Marie-Astrid Vernet, Gake Bouba, Serges Tchatchouang, Mohamadou Ripa Njankouo, Raphaël Tapondjou, Louis Deweerdt, Wilfred Mbacham, and Richard Njouom. Influence of meteorological parameters in the seasonality of influenza viruses circulating in northern cameroon. Influenza and other Respiratory Viruses, 13(2):158-165, Mar 2019. ISSN 1750-2659. doi: 10.1111/irv.12612.

[19] World Health Organization (WHO). World malaria report. 2018. ISBN 978-92-4-156565-3.

[20] Frank C Tanser, Brian Sharp, and David le Sueur. Potential effect of climate change on malaria transmission in Africa. The Lancet, 362(9398):1792 - 1798, 2003. ISSN 0140-6736. doi: https://doi.org/10.1016/S01406736(03)14898-2.

[21] Hector Caraballo and Kevin King. Emergency department management of mosquito-borne illness: malaria dengue, and west nile virus. Emergency medicine practice, 16(5):1-23, 2014

[22] Jonathan A. Patz and Sarah H. Olson. Malaria risk and temperature: influences from global climate change and local land use practices. Proceedings of the National Academy of Sciences of the United States of America, 103(15):5635-5636, Apr 2006. ISSN 0027-8424. doi: 10.1073/pnas.0601493103.

[23] David J. Rogers, Sarah E. Randolph, Robert W. Snow, and Simon I. Hay. Satellite imagery in the study and forecast of malaria. Nature, 415(6872):710-715, Feb 2002. ISSN 0028-0836. doi: 10.1038/415710a.

[24] S. I. Hay, C. J. Tucker, D. J. Rogers, and M. J. Packer. Remotely sensed surrogates of meteorological data for the study of the distribution and abundance of arthropod vectors of disease. Annals of Tropical Medicine Ef Parasitology, 90(1):1-19, 1996. doi: 10.1080/00034983.1996.11813021.

[25] Naoko Nihei, Yoshihiko Hashida, Mutsuo Kobayashi, and Akira Ishii. Analysis of malaria endemic areas on the indochina peninsula using remote sensing. Japanese Journal of Infectious Diseases, 55(5):160-166, Oct 2002. ISSN 1344-6304

[26] Mohammadreza Mobasheri and A. Sadeghi Naeini. Using IRS products to recover 7ETM+ defective images. American Journal of Applied Sciences, 5(6):618-625, 2007. doi: 10.3844/ajassp.2008.618.625.

[27] S. Liang. Quantitative Remote Sensing of Land Surfaces. Wiley Series in Remote Sensing and Image Processing. Wiley, 2004. ISBN 9780471723714

[28] W.G.M. Bastiaanssen, H. Pelgrum, J. Wang, Y. Ma, J.F. Moreno, G.J. Roerink, and T. van der Wal. A remote sensing surface energy balance algorithm for land (SEBAL).: Part 2: Validation. Journal of Hydrology, 212-213:213-229, 1998. ISSN 0022-1694. doi: https://doi.org/10.1016/S0022-1694(98)00254-6. URL http: //www.sciencedirect.com/science/article/pii/S0022169498002546. 
$\Longrightarrow$ Taylor \& Francis Taylor \& Francis Group

http://taylorandfrancis.com 